\title{
Transcatheter aortic valve implantation
}

\section{Background}

The aortic valve of the heart normally opens each time the heart contracts to allow the heart to pump oxygen-rich blood to the body's tissues. Narrowing of the aortic valve, known as aortic stenosis, occurs most commonly due to the age-related accumulation of calcium around the valve. This narrowing of the aortic valve obstructs the forward flow of blood from the heart and may result in severe fatigue, chest pain, shortness of breath, palpitations, dizziness and fainting, especially on exertion. Transcatheter aortic valve implantation (TAVI) or replacement (TAVR) is a minimally invasive procedure which serves as an alternative to surgical replacement of the aortic valve for those at high risk of surgical complications. This enables many high-risk individuals to benefit from alleviation of symptoms, an improved quality of life and prevention of serious long-term complications.

\section{Procedure}

TAVI involves insertion of a tissue replacement aortic valve which is mounted inside a stent of circular wire mesh and compressed into a small delivery tube or catheter. The procedure may be performed under sedation or a general anesthetic. Access to the aortic valve may be achieved in a number of ways. The most common approach, known as a transfemoral approach, involves insertion of the delivery tube into the femoral artery in the groin and, under continuous $\mathrm{X}$-ray imaging, the valve is threaded up the aorta to the original aortic valve. A transapical approach requires a horizontal incision to be made on the left side of the chest and the delivery system is inserted directly into the apex of the heart to access the site of the aortic valve. A transaortic approach involves an incision at the top of the breastbone with the delivery system inserted into the aorta. These alternative approaches may be necessary if peripheral arteries from the femoral artery to the aortic valve are too narrowed to pass the delivery tube and valve through. Once delivered to the aortic valve by any of these approaches, the metal stent is expanded and locked in place, pushing the original diseased valve to the side wall and allowing the new valve to function as a replacement valve. Individuals typically spend approximately a week in hospital following the procedure for close monitoring of their heart function.

\section{Benefits}

A TAVI procedure may significantly improve the symptoms and quality of life of those with severe aortic stenosis who are assessed as high-risk for more traditional surgical valve replacement via open heart surgery. TAVI may also prolong survival and decrease the risk of complications related to severe aortic stenosis, such as heart failure, abnormal heart rhythms and death.

\section{Risks}

As with all surgical procedures, TAVI carries a number of risks. While the risk of death is lower for individuals undergoing TAVI compared to those undergoing surgical valve replacements, the risk of stroke is higher. Other potential complications include wound infection, bleeding, leakage around the TAVI valve and the development of abnormal heart rhythms. As always, the risks should be weighed against the benefits and discussed with your surgeon. For more information, please visit the following websites: http://www.mayoclinic.org/tests-procedures/transcatheteraortic-valve-replacement/home/ovc-20204795

https://my.clevelandclinic.org/health/articles/transcatheteraortic-valve-replacement-tavr/tavr-procedure http://sydneyheartandlung.com.au/surgery/heart-surgery/ transcatheter-aortic-valve-implantation/

\begin{tabular}{ll} 
doi: $10.21037 /$ acs.2017.08.06 & For specific information concerning your medical condition, \\
\hline Section Writer: Christopher Harris & ACS suggests that you consult your physician. This page may be \\
photocopied non-commercially by physicians to share with patients. & Illustration Editor: Beth Croce
\end{tabular}

